Research Article

\title{
Multiobjective Optimization of Nonlinear Active Suspension System with Time-Delayed Feedback
}

\author{
Su-Juan Shao $\mathbb{D}^{1},{ }^{1}$ Dong Jing $\mathbb{D}^{2},{ }^{2}$ and Chuan-Bo Ren $\mathbb{D}^{2}$ \\ ${ }^{1}$ School of Mechanical Engineering, Shandong University of Technology, Zibo, Shandong, China \\ ${ }^{2}$ School of Transportation and Vehicle Engineering, Shandong University of Technology, Zibo, Shandong, China \\ Correspondence should be addressed to Chuan-Bo Ren; chuanbor@sdut.edu.cn
}

Received 2 May 2020; Revised 24 July 2020; Accepted 21 September 2020; Published 5 November 2020

Academic Editor: Georgios I. Giannopoulos

Copyright (C) 2020 Su-Juan Shao et al. This is an open access article distributed under the Creative Commons Attribution License, which permits unrestricted use, distribution, and reproduction in any medium, provided the original work is properly cited.

Considering the nonlinear properties of spring and damping of suspension, a quarter-car model with time-delayed control is established. The Routh-Hurwitz stability criterion and stability switching method are used to analyze the stability of the system and obtain the stability region diagram. The multiobjective optimization function is established by considering the ride comfort, driving safety, and handling stability. The optimal control parameters are obtained by the optimization and simulation of the system under harmonic excitation and random excitation. In addition, the responses of the active suspension system with optimal time-delay control and the passive suspension system without control are compared. The results show that the active suspension system with time-delay displacement feedback control can reduce the vibration of the system, and there is an optimal feedback parameter combination to optimize the vehicle running state. The design of multiobjective function optimization proposed in this paper can improve ride comfort, driving safety, and handling stability and provide guidance for comprehensively improving vehicle performance.

\section{Introduction}

The suspension system is an important assembly connecting the wheel and the body, which transfers the force and torque between the wheel and the body. The reasonable design of the automobile suspension system can improve the ride performance, handling stability, and ride comfort. At present, the majority of automobile suspension is passive suspension, in which suspension parameters cannot be adjusted once selected. Therefore, the optimal performance can only be achieved at a certain speed and road conditions, and the ability to adapt to different road conditions is still weak. Since the concept of active control was introduced into the field of vehicle engineering in the 1960s, research on the semiactive and active suspension system has received extensive attention. For the study of the active suspension system, linear quadratic optimal control principle [1] and classical PID control [2] based on the linear model were adopted in the early stage. The time-delay method is derived from the concept of the time-delay dynamic absorber proposed by Olgac et al. [3] in 1992. The time-delay vibration reduction control method based on displacement was used to control the single-frequency harmonic excitation system, and better damping effect was obtained. Subsequently, Olgac et al. $[4,5]$ carried out further studies on the time-delay dynamic absorber. The single-degree-of-freedom system and the multidegree-of-freedom system as well as the dual delay and dual frequency excitation were studied. Compared with the passive absorber, the time-delay dynamic absorber had better damping effect and wider frequency band. With the introduction of timedelay vibration reduction theory, a large number of scholars have applied this theory to vibration reduction control in different fields. Alhazza et al. [6] used the time-delay vibration reduction method to control the vibration reduction of the cantilever beam and obtained a good vibration reduction effect. Liu et al. [7] carried out theoretical and experimental studies on the time-delay state feedback control technology with flexible beams as the research object, and the results showed that reasonable selection of time delay could effectively reduce the vibration of flexible beams. Wang et al. [8] carried out the twotime-delay feedback control for the five-degree-of-freedom half- 
car model, and the results showed that the time-delay feedback control based on engine acceleration in the idle condition significantly improved the vibration damping performance of the suspension system compared with the uncontrolled condition. This kind of vibration damping technology has been applied to many engineering fields, among which the research of the time-delay active control suspension system and its related research has become a hotspot in recent years. Compared with other control methods of active suspension, time-delay feedback control has the characteristics of simple algorithm and less computation of the control law.

In order to simplify the mathematical model of the complex vehicle system, many scholars have carried out a lot of theoretical analysis and experimental research. Considering that both the elastic elements and the damping elements have different degrees of nonlinear characteristics, Du et al. $[9,10]$ applied the nonfragile $H \infty$ robust control method to the nonlinear mechanical vibration system. Zhou et al. [11] used quadratic and cubic functions to describe the nonlinear uncertainty of vehicle suspension and tires. Koumene Taffo et al. [12] used quadratic and cubic terms to characterize the nonlinear characteristics of a suspension spring when establishing the nonlinear (1/4) vehicle model. In the study of the timedelay nonlinear dynamic vibration absorber, Zhao and $\mathrm{Xu}$ [13] used cubic polynomial to represent the nonlinear uncertainty of a spring.

It is found that time delay not only hinders the operation of the system but also often leads to the generation of limit cycles, loss of stability, and bifurcation chaos. In particular, the dynamic behavior of the system is complicated due to time delay and nonlinear coupling. However, the appropriate feedback control law can improve the system performance and even stabilize the unstable system [14]. Some studies have shown that the time delay can make the system have the optimal dynamic behavior [15]. Therefore, it is necessary to study the influence of time delay on system stability and dynamic behavior. However, for linear time-invariant systems with time delay, the characteristic equations become transcendent due to the exponential functions associated with time delay. Transcendence brings about an infinite number of characteristic roots, which are very troublesome to deal with [16]. In order to solve this problem, many methods have been proposed to evaluate the stability of time-delay systems, such as the generalized Sturm criterion method [17] and the D-subdivision method [18] and its improved version [19]. $\mathrm{Xu}$ and $\mathrm{Lu}$ [20] studied the influence of time-delay feedback control on the dynamic behavior of nonlinear systems with periodic external excitation. Through numerical analysis, it was observed that time delay could lead to instability of the static and dynamic equilibrium states of the system, resulting in Hopf bifurcation, period-doubling bifurcation, almost periodic motion, chaos, and other motion behaviors. Koumene Taffo et al. [12] studied a class of two-degreeof-freedom nonlinear quarter-car models with time-delay feedback control. The results showed that the system had a small time-delay-independent stability region. When the system was not in the time-delay-independent region, stability switch might occur with the change of the time delay. These admissible switches corresponded to Hopf bifurcation when the time delay crossed the critical value, and the properties of periodic solution direction and stability of Hopf bifurcation were determined by using normal form theory and central manifold theorem. Wang et al. [21] added time-delay speed feedback control to the maglev train system, studied the stability and bifurcation of the system based on the central manifold, and showed that when the time delay exceeded a certain value, the system would produce Hopf bifurcation.

In recent years, the time-delay vibration reduction technology has been further developed, and its application in the engineering field is becoming more and more extensive. In the study of vibration damping performance of the time-delay control system, the superiority of time-delay control is demonstrated by comparing the vibration effect of the main system before and after adding time-delay control, and the form of excitation is generally selected as harmonic excitation, which is very different from the complex and changeable road conditions. In addition, in the optimal design of the time-delay control system, the absolute value of the frequency response of the displacement at the resonance point is selected as the objective function, which is independent of the excitation. The factors that directly affect the performance of the vehicle are not considered, such as acceleration of the vehicle body, dynamic deflection of the suspension, and dynamic displacement of the tire.

The selection of appropriate feedback parameters can effectively reduce the vibration, so the optimal time-delay feedback control design has become a research focus. Huan et al. [22] considered three contradictory objective functions: peak frequency response function, main structure vibration energy function, and control work function and gave the Pareto optimal solution, which was validated by numerical analysis. Liu et al. [23] proposed an optimal time-delay feedback control method with attenuation ratio as the objective function to reduce the vibration of flexible simply supported beams under the main resonance and ultraharmonic resonance. Through the simulation analysis, the piezoelectric optimal controller is designed to obtain a better vibration control effect. Li et al. [24] took the sum of the frequency domain response functions of the vibration displacement, velocity, and acceleration of the main system as the objective function when optimizing the timevarying parameter of the time-delay dynamic vibration absorber.

In this paper, the theory of time-delay vibration reduction is applied to the automobile suspension system. A time-delay feedback control force based on vehicle displacement is applied to the system to realize the active control of the nonlinear suspension system to achieve the purpose of vibration reduction. The main contributions of this paper are summarized as follows:

(i) Considering the nonlinear uncertainty of spring force and damping force of suspension, a nonlinear active suspension system model with time delay is established.

(ii) Generalized Sturm criterion and Routh-Hurwitz stability theorem are used to analyze the stability of the system. The time-delay-independent stability interval and critical time-delay curve are obtained to ensure the robustness of the system. 


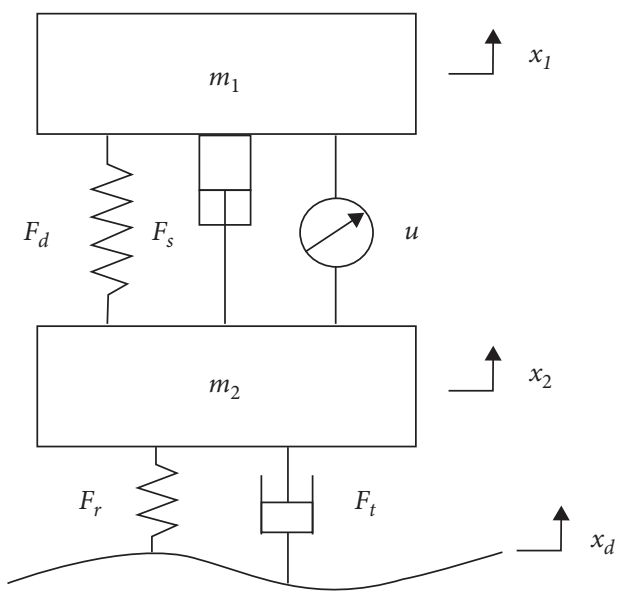

FIgURE 1: Model of the (1/4) active suspension system.

(iii) A multiobjective optimization control method is proposed, which considers the car body acceleration, the suspension dynamic deflection, and the tire dynamic displacement. In addition, the optimization results can comprehensively improve the vehicle performance.

(iv) Simulation analysis of the system under simple harmonic excitation and random excitation is carried out, and the conclusion is more persuasive.

\section{Nonlinear Active Suspension System Model}

2.1. Modeling. Considering the nonlinear factors of elastic element and damping element, the nonlinear two-degree-offreedom model of the $(1 / 4)$ vehicle is established, as shown in Figure 1 , where $m_{1}$ is sprung mass, $m_{2}$ is unsprung mass, $F_{s}$ is damping force, $F_{d}$ is spring elastic force, $u$ is actuator control force, $F_{r}$ is tire elastic force, and $F_{t}$ is tire damping force. $x_{1}, x_{2}$, and $x_{d}$ are sprung mass displacement (i.e., body displacement), unsprung mass displacement (i.e., tire displacement), and road excitation displacement, respectively.

According to Newton's second law, the dynamic equations of the $(1 / 4)$ vehicle active suspension system can be expressed as follows:

$$
\begin{array}{r}
m_{1} \ddot{x}_{1}+F_{s}+F_{d}+u=0, \\
m_{2} \ddot{x}_{2}-F_{s}-F_{d}+F_{r}+F_{t}-u=0,
\end{array}
$$

where

$$
\begin{aligned}
F_{d} & =k_{1}\left(x_{1}-x_{2}\right)+\alpha_{1}\left(x_{1}-x_{2}\right)^{3}, \\
F_{s} & =c_{1}\left(\dot{x}_{1}-\dot{x}_{2}\right)+\alpha_{2}\left(\dot{x}_{1}-\dot{x}_{2}\right)^{3}, \\
F_{r} & =k_{2}\left(x_{2}-x_{d}\right), \\
F_{t} & =c_{2}\left(\dot{x}_{2}-\dot{x}_{d}\right), \\
u & =g_{1}\left[x_{1}(t-\tau)-x_{1}(t)\right],
\end{aligned}
$$

where $k_{1}$ and $\alpha_{1}$ are the linear and nonlinear stiffness coefficients of the spring, respectively, $c_{1}$ and $\alpha_{2}$ are linear and nonlinear damping coefficients of suspension damping, respectively, $k_{2}$ and $c_{2}$ are the stiffness coefficient and the damping coefficient of the tire, respectively, and $g_{1}$ and $\tau$ are the feedback gain coefficient and time delay, respectively. When $\tau=0$, the actuator control force $u$ is zero, and the system becomes a passive suspension system without timedelayed feedback control. By substituting $F_{s}, F_{d}, F_{r}$, $F_{t}$, and $u$ into equations (1) and (2), we obtain the following equations:

$$
m_{1} \ddot{x}_{1}+k_{1}\left(x_{1}-x_{2}\right)+\alpha_{1}\left(x_{1}-x_{2}\right)^{3}+c_{1}\left(\dot{x}_{1}-\dot{x}_{2}\right)+\alpha_{2}\left(\dot{x}_{1}-\dot{x}_{2}\right)^{3}+g_{1}\left[x_{1}(t-\tau)-x_{1}(t)\right]=0
$$

$m_{2} \ddot{x}_{2}-k_{1}\left(x_{1}-x_{2}\right)-\alpha_{1}\left(x_{1}-x_{2}\right)^{3}-c_{1}\left(\dot{x}_{1}-\dot{x}_{2}\right)-\alpha_{2}\left(\dot{x}_{1}-\dot{x}_{2}\right)^{3}+k_{2}\left(x_{2}-x_{d}\right)+c_{2}\left(\dot{x}_{2}-\dot{x}_{d}\right)-g_{1}\left[x_{1}(t-\tau)-x_{1}(t)\right]=0$. 
2.2. Nondimensionalizing. To simplify the analysis, $t$ and $\tau$ are written in dimensionless form as follows:

$$
\begin{aligned}
t^{*} & =\sqrt{\frac{k_{1}}{m_{1}}} t, \\
\tau^{*} & =\sqrt{\frac{k_{1}}{m_{1}}} \tau .
\end{aligned}
$$

Dimensionless parameters are introduced as follows:

$$
\begin{aligned}
& x_{1}^{*}=\frac{x_{1}}{L}, \\
& x_{2}^{*}=\frac{x_{2}}{L}, \\
& x_{d}^{*}=\frac{x_{d}}{L}, \\
& \mu=\frac{\alpha_{1} L^{2}}{\mathrm{k}_{1}}, \\
& \delta=\frac{\alpha_{2} L^{2} \sqrt{\mathrm{k}_{1}}}{\sqrt{m_{1}^{3}}}, \\
& \zeta_{1}=\frac{c_{1}}{\sqrt{m_{1} \mathrm{k}_{1}}}, \\
& \zeta_{2}=\frac{c_{2}}{\sqrt{m_{1} \mathrm{k}_{1}}}, \\
& g=\frac{g_{1}}{k_{1}}, \\
& k=\frac{m_{2}}{k_{1}}, \\
& m_{2}
\end{aligned}
$$

where $L$ is the unit length, in order to make the formula dimensionless. For the sake of writing convenience, $x_{1}^{*}, x_{2}^{*}, x_{d}^{*}, t^{*}$, and $\tau^{*}$ are written as $x_{1}, x_{2}, x_{d}, t$, and $\tau$. Let $x_{1 \tau}=x_{1}^{*}(t-\tau)$.

$$
\begin{aligned}
\ddot{x}_{1} & +\left(x_{1}-x_{2}\right)+\mu\left(x_{1}-x_{2}\right)^{3}+\zeta_{1}\left(\dot{x}_{1}-\dot{x}_{2}\right) \\
& +\delta\left(\dot{x}_{1}-\dot{x}_{2}\right)^{3}+g x_{1 \tau}-g x_{1}=0 \\
\ddot{x}_{2} & -\gamma\left(x_{1}-x_{2}\right)-\gamma \mu\left(x_{1}-x_{2}\right)^{3}-\gamma \zeta_{1}\left(\dot{x}_{1}-\dot{x}_{2}\right) \\
& -\gamma \delta\left(\dot{x}_{1}-\dot{x}_{2}\right)^{3}+\gamma k\left(x_{2}-x_{d}\right)+\gamma \zeta_{2}\left(\dot{x}_{2}-\dot{x}_{d}\right) \\
& -\gamma g x_{1 \tau}+\gamma g x_{1}=0 .
\end{aligned}
$$

Equations (8) and (9) are reduced to first-order differential equations of motion:

$$
\begin{aligned}
\dot{x}_{1}= & y_{1}, \\
\dot{y}_{1}= & -\left(x_{1}-x_{2}\right)-\mu\left(x_{1}-x_{2}\right)^{3}-\zeta_{1}\left(y_{1}-y_{2}\right)-\delta\left(y_{1}-y_{2}\right)^{3} \\
& -g x_{1 \tau}+g x_{1}, \\
\dot{x}_{2}= & y_{2}, \\
\dot{y}_{2}= & \gamma\left(x_{1}-x_{2}\right)+\gamma \mu\left(x_{1}-x_{2}\right)^{3}+\gamma \zeta_{1}\left(y_{1}-y_{2}\right) \\
& +\gamma \delta\left(y_{1}-y_{2}\right)^{3}-\gamma k\left(x_{2}-x_{d}\right)-\gamma \zeta_{2}\left(y_{2}-\dot{x}_{d}\right) \\
& +\gamma g x_{1 \tau}-\gamma g x_{1} .
\end{aligned}
$$

2.3. Stability Analysis. In engineering problems, steady-state motion usually corresponds to the state in which the machine can work normally. The stability of equilibrium state or periodic motion can change with the change of system parameters, resulting in bifurcation or chaotic motion. The stability of the system is analyzed to ensure that the results of optimization parameters meet the stability conditions of the system. When the system has no external excitation, the stability of the static equilibrium state directly determines the stability of the system under excitation. The associated autonomous linear system of equation (10) is as follows:

$$
\begin{aligned}
& \dot{x}_{1}=y_{1}, \\
& \dot{y}_{1}=-\left(x_{1}-x_{2}\right)-\zeta_{1}\left(y_{1}-y_{2}\right)-g x_{1 \tau}+g x_{1}, \\
& \dot{x}_{2}=y_{2}, \\
& \dot{y}_{2}=\gamma\left(x_{1}-x_{2}\right)+\gamma \zeta_{1}\left(y_{1}-y_{2}\right)-\gamma k x_{2}-\gamma \zeta_{2} y_{2}+\gamma g x_{1 \tau}-\gamma g x_{1} .
\end{aligned}
$$

Defining new variables $\mathbf{x}=\left[x_{1}, y_{1}, x_{2}, y_{2}\right]^{T}$ and $\mathbf{x}_{\tau}=\left[x_{1 \tau}, y_{1 \tau}, x_{2 \tau}, y_{2 \tau}\right]^{T}$, equation (11) becomes

$$
\dot{x}=\mathbf{A}_{1} \mathbf{x}+\mathbf{A}_{2} \mathbf{x}_{\tau},
$$

where

$$
\begin{aligned}
\mathbf{A}_{1} & =\left[\begin{array}{cccc}
0 & 1 & 0 & 0 \\
-1+g & -\zeta_{1} & 1 & \zeta_{1} \\
0 & 0 & 0 & 1 \\
\gamma-\gamma g & \gamma \zeta_{1} & -\gamma-\gamma k & -\gamma \zeta_{1}-\gamma \zeta_{2}
\end{array}\right], \\
\mathbf{A}_{2} & =\left[\begin{array}{cccc}
0 & 0 & 0 & 0 \\
-g & 0 & 0 & 0 \\
0 & 0 & 0 & 0 \\
\gamma g & 0 & 0 & 0
\end{array}\right] .
\end{aligned}
$$

According to the theory of hysteretic ordinary differential equations, differential equation (12) has formal solution: $\mathbf{x}=\mathbf{x}_{0} e^{\lambda t}$, and substituting it into equation (12), we obtain the following characteristic equation:

$$
\operatorname{det}\left(\lambda I-\mathbf{A}_{1}-\mathbf{A}_{2} e^{-\lambda \tau}\right)=0,
$$


where $\lambda$ is the characteristic root of the characteristic equation (14). The characteristic equation (14) is expanded into a polynomial form:

$$
\mathrm{D}(\lambda, \tau)=P(\lambda)+Q(\lambda) e^{-\lambda \tau}=0,
$$

where $P(\lambda)$ and $Q(\lambda)$ are real coefficient polynomials of fourth and second order, respectively:

$$
\begin{aligned}
& \mathrm{P}(\lambda)=\lambda^{4}+p_{3} \lambda^{3}+p_{2} \lambda^{2}+p_{1} \lambda+p_{0}, \\
& \mathrm{Q}(\lambda)=q_{2} \lambda^{2}+q_{1} \lambda+q_{0},
\end{aligned}
$$

where

$$
\begin{aligned}
& p_{0}=\gamma k-g \gamma k, \\
& p_{1}=\zeta_{1} \gamma k+\gamma \zeta_{2}-g \gamma \zeta_{2}, \\
& p_{2}=\gamma \zeta_{1} \zeta_{2}+\gamma k+\gamma+1-g, \\
& p_{3}=\gamma \zeta_{1}+\zeta_{1}+\gamma \zeta_{2}, \\
& q_{0}=g \gamma k, \\
& q_{1}=g \gamma \zeta_{2}, \\
& q_{2}=g,
\end{aligned}
$$

and when $\tau=0$, the characteristic polynomial equation (15) becomes

$$
D(\lambda, \tau)=\lambda^{4}+p_{3} \lambda^{3}+\left(p_{2}+q_{2}\right) \lambda^{2}+\left(p_{1}+q_{1}\right) \lambda+p_{0}+q_{0} .
$$

The Routh-Hurwitz stability conditions of the passive suspension system without time delay are shown as follows:

$$
\begin{aligned}
& R_{1}=p_{3}>0, \\
& R_{2}=p_{2}+q_{2}>0, \\
& R_{3}=p_{1}+q_{1}>0, \\
& R_{4}=p_{0}+q_{0}>0, \\
& R_{5}=p_{3}\left(p_{2}+q_{2}\right)\left(p_{1}+q_{1}\right)-\left(p_{1}+q_{1}\right)^{2}-p_{3}^{2}\left(p_{0}+q_{0}\right)>0 .
\end{aligned}
$$

When $\tau>0$, according to the first-order approximation stability theory for nonlinear systems [25], when all the real parts of the first-order approximation equation are negative, the equilibrium solution of the original nonlinear equation is asymptotically stable. Then, the critical condition for the instability of the system is that the characteristic equation has a pure imaginary root $\lambda=i \omega$. By substituting it into equation (15), substituting with Euler's formula: $e^{-\lambda \tau}=e^{-i \omega \tau}=\cos (\omega \tau)-i \sin (\omega \tau)$, separating the real and imaginary parts of the equation, and setting them equal to zero, respectively, the following equation can be obtained:

$$
\begin{aligned}
& P_{R}(\omega)+Q_{R}(\omega) \cos (\omega \tau)+Q_{I}(\omega) \sin (\omega \tau)=0, \\
& P_{I}(\omega)-Q_{R}(\omega) \sin (\omega \tau)+Q_{I}(\omega) \cos (\omega \tau)=0,
\end{aligned}
$$

where

$$
\begin{aligned}
P_{R}(\omega)= & \operatorname{Re}[P(i \omega)]=\omega^{4}-\left(\gamma \zeta_{1} \zeta_{2}+\gamma k+\gamma+1-g\right) \omega^{2} \\
& +\gamma k-g \gamma k, \\
P_{I}(\omega)= & \operatorname{Im}[P(i \omega)]=-\left(\gamma \zeta_{1}+\zeta_{1}+\gamma \zeta_{2}\right) \omega^{3} \\
& +\left(\zeta_{1} \gamma k+\gamma \zeta_{2}-g \gamma \zeta_{2}\right) \omega, \\
Q_{R}(\omega)= & \operatorname{Re}[Q(i \omega)]=-g \omega^{2}+g \gamma k, \\
Q_{I}(\omega)= & \operatorname{Im}[Q(i \omega)]=g \gamma \zeta_{2} \omega .
\end{aligned}
$$

By eliminating the trig terms, we get the equation as follows:

$$
P_{R}(\omega)^{2}+P_{I}(\omega)^{2}-\left[Q_{R}(\omega)^{2}+Q_{I}(\omega)^{2}\right]=0 .
$$

then,

Let $\quad F(\omega)=P_{R}(\omega)^{2}+P_{I}(\omega)^{2}-\left[Q_{R}(\omega)^{2}+Q_{I}(\omega)^{2}\right] ;$

$$
F(\omega)=\omega^{8}+b_{1} \omega^{6}+b_{2} \omega^{4}+b_{3} \omega^{2}+b_{4},
$$

where

$$
\begin{aligned}
b_{1}= & -2 \gamma \zeta_{1} \zeta_{2}-2 \gamma k-2 \gamma+2 g-2+\left(-\gamma \zeta_{1}-\zeta_{1}-\gamma \zeta_{2}\right)^{2}, \\
b_{2}= & 2 \gamma k-2 g \gamma k+\left(-\gamma \zeta_{1} \zeta_{2}-\gamma k+g-\gamma-1\right)^{2} \\
& +2\left(\zeta_{1} \gamma k+\gamma \zeta_{2}-g \gamma \zeta_{2}\right)\left(-\gamma \zeta_{1}-\zeta_{1}-\gamma \zeta_{2}\right)-g^{2}, \\
b_{3}= & 2(\gamma k-g \gamma k)\left(-\gamma \zeta_{1} \zeta_{2}-\gamma k+g-\gamma-1\right) \\
& +\left(\zeta_{1} \gamma k+\gamma \zeta_{2}-g \gamma \zeta_{2}\right)^{2}+2 g^{2} \gamma k-g^{2} \gamma^{2} \zeta_{2}^{2}, \\
b_{4}= & (\gamma k-g \gamma k)^{2}-g^{2} \gamma^{2} k^{2} .
\end{aligned}
$$

By calculating equation (20), we can get that $\omega \tau$ satisfies a set of trigonometric function equations as follows:

$$
\begin{aligned}
& \cos (\omega \tau)=-\frac{P_{R}(\omega) Q_{R}(\omega)+P_{I}(\omega) Q_{I}(\omega)}{Q_{R}(\omega)^{2}+Q_{I}(\omega)^{2}}, \\
& \sin (\omega \tau)=\frac{P_{I}(\omega) Q_{R}(\omega)-P_{R}(\omega) Q_{I}(\omega)}{Q_{R}(\omega)^{2}+Q_{I}(\omega)^{2}} .
\end{aligned}
$$

From equations (23) and (25), we can get the function relations of $g$ and $\tau$ with respect to $\omega$, respectively: $g=G(\omega)$ and $\tau=\mathrm{T}(\omega)$. According to the parameter equations, the critical stability curves of $g$ and $\tau$ are obtained, as shown in Figure 2. The parameters of the model are selected as follows: $m_{1}=320 \mathrm{~kg}, m_{2}=40 \mathrm{~kg}, k_{1}=18000(\mathrm{~N} / \mathrm{m}), k_{2}=$ $200000(\mathrm{~N} / \mathrm{m}), \quad c_{1}=1200\left(\mathrm{Ns} / \mathrm{m}, \quad c_{2}=4000(\mathrm{Ns} / \mathrm{m}, \quad\right.$ and $g_{1}=-20000 \sim 20000(\mathrm{~N} / \mathrm{m})$. Then, we get the parameters $\zeta_{1}=0.5, \quad \zeta_{2}=1.667, \quad \gamma=8, \quad k=11.111, \quad$ and $g \in[-1.111,1.111]$. By calculation, the Routh-Hurwitz stability conditions (equation (19)) are unconditionally stable, which means that the corresponding nondelay system of the active suspension system with time delay is Routh-Hurwitz stable. According to Figure 2, when $g$ falls roughly in the interval $(-0.35,0.35)$, the system is timeindependent stable, that is, the system is unconditionally 


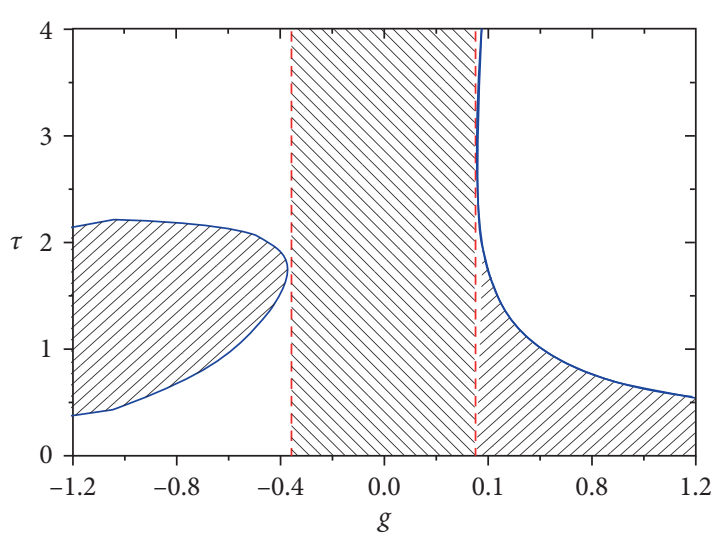

FIgure 2: Curve of critical time delay.

stable in this interval. When $g$ falls into other regions, as the time delay increases, the system undergoes several stabilization switches and eventually becomes unstable [6]. These admissible switches correspond to the Hopf bifurcation that occurs when the delay spans the critical value. The shaded parts are the stable regions.

\section{Optimization and Simulation}

3.1. Optimization and Simulation under Sinusoidal Excitation. Assuming that the system is under the action of periodic simple harmonic pavement excitation $x_{d}=$ $A \sin (\omega t)$, which is substituted into equation (10), the dimensionless equation is

$$
\begin{aligned}
\dot{x}_{1}= & y_{1}, \\
\dot{y}_{1}= & -\left(x_{1}-x_{2}\right)-\mu\left(x_{1}-x_{2}\right)^{3}-\zeta_{1}\left(y_{1}-y_{2}\right) \\
& -\delta\left(y_{1}-y_{2}\right)^{3}-g x_{1 \tau}+g x_{1}, \\
\dot{x}_{2}= & y_{2}, \\
\dot{y}_{2}= & \gamma\left(x_{1}-x_{2}\right)+\gamma \mu\left(x_{1}-x_{2}\right)^{3}+\gamma \zeta_{1}\left(y_{1}-y_{2}\right) \\
& +\gamma \delta\left(y_{1}-y_{2}\right)^{3}-\gamma k\left[x_{2}-A^{*} \sin (\Omega t)\right] \\
& -\gamma \zeta_{2}\left[y_{2}-A^{*} \Omega \cos (\Omega t)\right]+\gamma g x_{1 \tau}-\gamma g x_{1},
\end{aligned}
$$

where $\Omega=\sqrt{m_{1} / k_{1} \omega}$ and $A^{*}=(A / L)$.

3.1.1. The Objective Function. Since acceleration is a direct response of vibration energy, the value of automobile acceleration directly reflects the ride comfort of passengers. According to the international standard ISO2631, the weighted sum of the root mean square value of vibration acceleration of each degree of freedom is used as the evaluation index of vehicle smoothness. The model in this paper is a two-degree-of-freedom one-quarter vehicle model, and the root mean square value of vertical acceleration is used as the evaluation index of vehicle smoothness. The dynamic deflection of the suspension represents the relative displacement between the body and the tires, and its value should not be too large, or the limit block in the process of driving will often be hit and even lead to the breakdown of the suspension, affecting the driving safety of the car. In addition, the dynamic load of the tire should not be too large. Too much dynamic load will affect the adhesion effect of tires and even cause tires to jump off the ground. Because the damping force of the tire is small compared with the spring force, the dynamic displacement of the tire is used to represent the dynamic load of the tire in this paper. Take ride comfort, driving safety, and handling stability as the main performance indicators to establish the objective function. Considering that the car body acceleration, suspension dynamic deflection, and tire dynamic displacement are different in order of magnitude, the weighted sum of the ratio of their RMS values is taken as the objective function. According to the importance of each indicator, the weighted coefficients of the three factors are $0.6,0.2$, and 0.2 , respectively. The objective function is shown in equation (27). The constraint conditions are shown in equation (28).

$$
\begin{aligned}
& a=0.6 \frac{\ddot{x}_{1 \mathrm{rms}}}{\ddot{x}_{1 \mathrm{rms}}^{\prime}}+0.2 \frac{x_{\alpha \mathrm{rms}}}{x_{\alpha \mathrm{rms}}^{\prime}}+0.2 \frac{x_{\beta \mathrm{rms}}}{x_{\beta \mathrm{rms}}^{\prime}}, \\
& \text { s.t. }\left\{\begin{array}{l}
-1.11 \leq g \leq 1.11, \\
0<\tau \leq 10,
\end{array}\right.
\end{aligned}
$$

where $\ddot{x}_{1 \mathrm{rms}}^{\prime}, x_{\alpha \mathrm{rms}}^{\prime}$, and $x_{\beta \mathrm{rms}}^{\prime}$ are the RMS values of the car body acceleration, the suspension dynamic deflection, and the tire dynamic displacement, respectively, of the passive suspension. $\ddot{x}_{1 \mathrm{rms}}, x_{\alpha \mathrm{rms}}$, and $x_{\beta \mathrm{rms}}$ are the RMS values of the car body acceleration, suspension dynamic deflection, and tire dynamic displacement, respectively, of the active suspension with time-delay feedback control.

3.1.2. Optimization and Simulation. Taking the minimum value of the objective function equation (27) as the optimization condition, with equation (28) as the constraint conditions, the optimal feedback parameters are obtained. Particle swarm optimization is used for optimization; the step lengths of $g$ and $\tau$ are set to 0.01 . The parameters values of the model are $\alpha_{1}=180\left(\mathrm{~N} / \mathrm{m}^{3}\right), \alpha_{2}=20\left(\mathrm{Ns}^{3} / \mathrm{m}^{3}\right)$, $A=0.05$, and $\omega=\sqrt{k_{1} / m_{1}}$; the corresponding dimensionless parameters values are $\mu=0.01, \delta=0.469, A^{*}=0.05$, and $\Omega=1$. The initial condition is $\left[x_{1}(t) y_{1}(t)\right.$ $\left.x_{2}(t) y_{2}(t)\right]=\left[\begin{array}{llll}0 & 0 & 0 & 0\end{array}\right]$, where $t \in[-\tau, 0]$. The optimization results are $g=-1.03$ and $\tau=2.07$. It can be seen from Figure 2 that the optimization results meet the stability conditions. The corresponding parameters of the original model are $g_{1}=-18540(\mathrm{~N} / \mathrm{m})$ and $\tau=0.276 \mathrm{~s}$. In order to verify the correctness of the optimization results, the dynamic responses of the active suspension system under the optimal control parameters are compared with those of the passive suspension system without control. Mathematical software Matlab is used for the simulation calculation of equations (4) and (5), and the simulation figures are shown in Figure 3.

According to Figure 3, it can be found that the acceleration amplitude of the car body, suspension dynamic deflection amplitude, and tire dynamic displacement amplitude under the optimal control parameters are far less than those under the passive suspension system without 


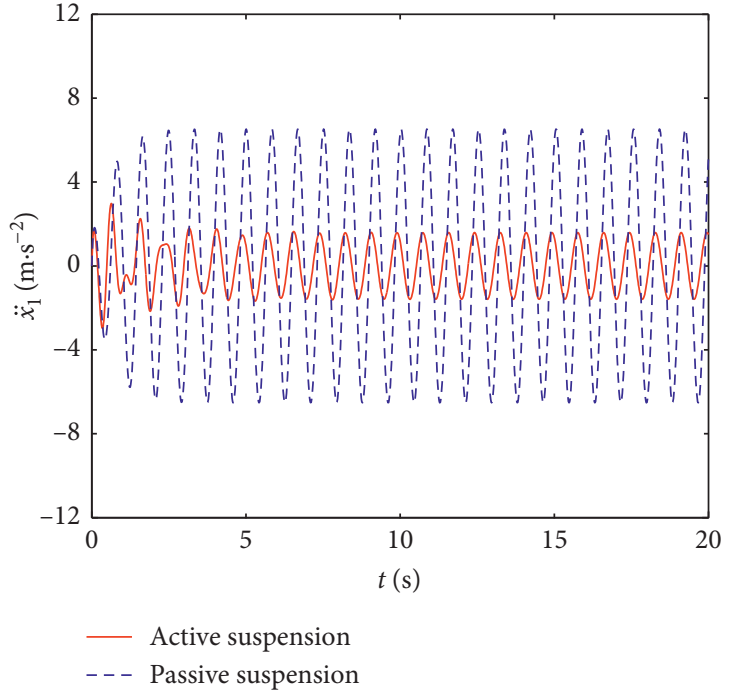

(a)

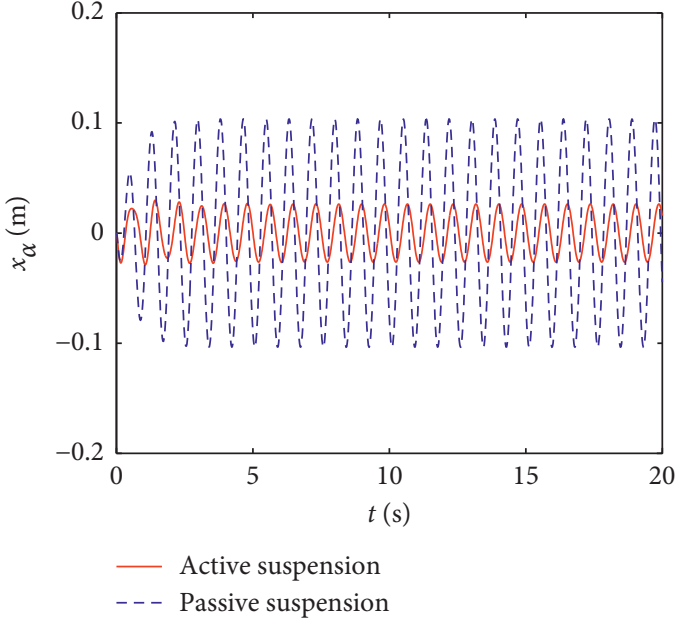

(b)

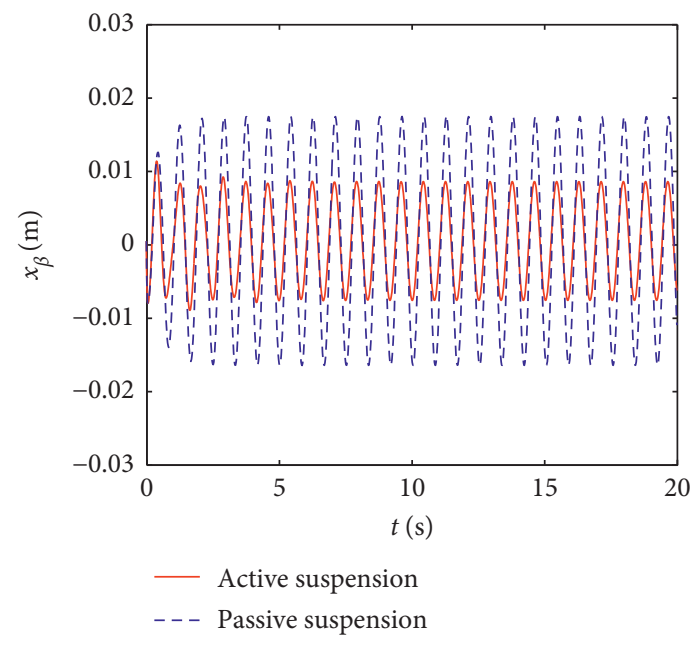

(c)

FIgURE 3: Simulation results. (a) Acceleration of the car body. (b) Suspension dynamic deflection. (c) Tire dynamic displacement.

control. The value of the vertical acceleration of the vehicle body directly reflects the ride comfort of passengers, so adding the time-delay feedback controller can greatly improve ride comfort. The reduction of the amplitudes of the suspension dynamic deflection and the tire dynamic displacement means better driving safety and better grasping performance. The RMS values of the system responses under optimal parameters control and without control are calculated, and the damping effect of time-delay feedback control is analyzed quantitatively, and the results are listed in Table 1 . The RMS values of car body acceleration, suspension dynamic deflection, and tire dynamic displacement are reduced by $70.7925 \%, 69.4882 \%$, and $51.8993 \%$, respectively.

\section{Optimization and Simulation under Random Excitation}

4.1. Dimensionless Model. From the above analysis, it can be seen that under simple harmonic excitation, the active suspension system with time-delay feedback control has achieved better vibration control effect compared with the passive suspension system without control. The actual road surface is not the periodic harmonic excitation under the ideal state. In order to get closer to the real state, the performance of the active suspension system with time-delay feedback control is further studied. Assume that the system is affected by random road excitation $x_{d}=\mathrm{B} \cdot \mathrm{gs}(t)$, where 
TABLE 1: RMS of the responses.

Sinusoidal excitation

Passive suspension

Active suspension under optimal parameters

Percentage change
Acceleration of the car body Suspension dynamic deflection Tire dynamic displacement

$\begin{array}{ccc}4.3056 & 0.0658 & 0.0110 \\ 1.2576 & 0.0201 & 0.0053 \\ -70.7925 \% & -69.4882 \% & -51.8993 \%\end{array}$

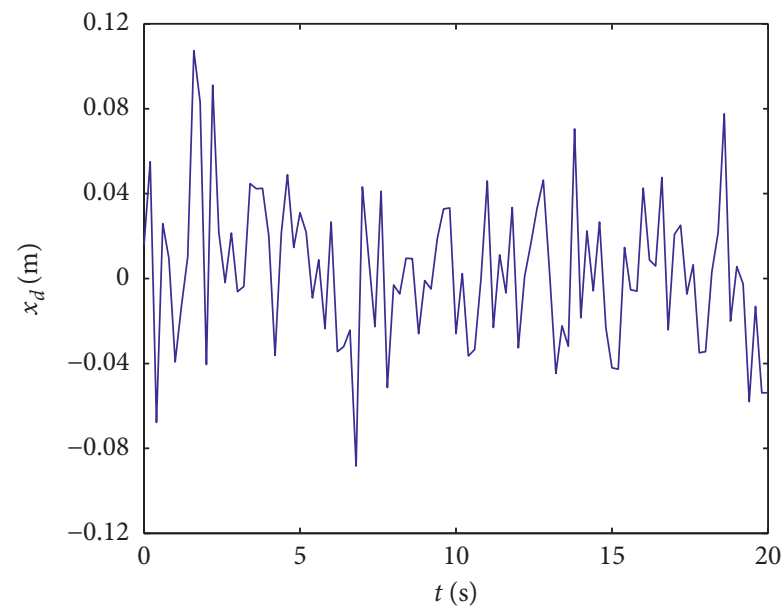

FIgURE 4: Time-domain diagram of random excitation.

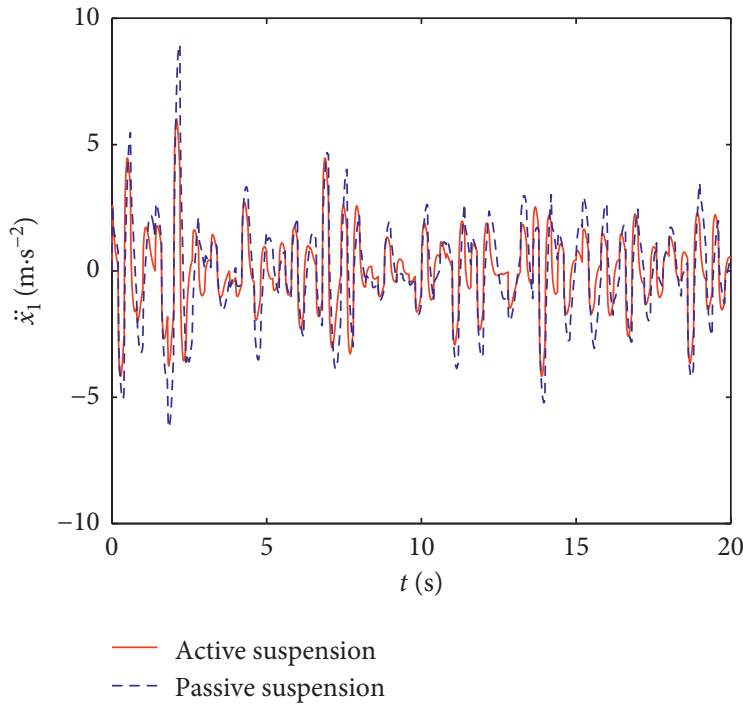

(a)

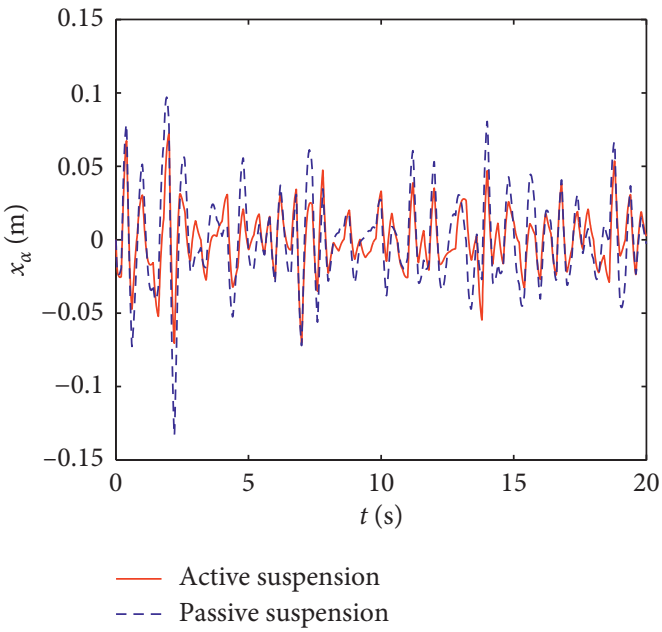

(b)

FIGURE 5: Continued. 


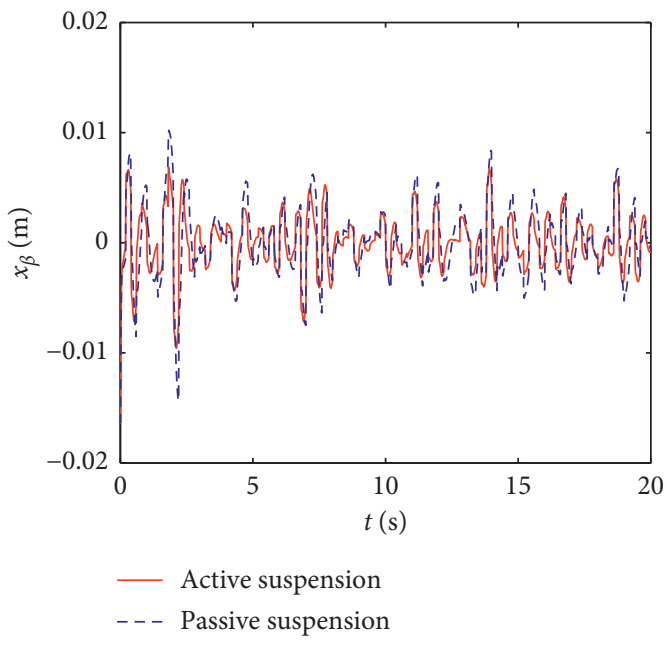

(c)

FIgURE 5: Simulation results. (a) Acceleration of the car body. (b) Suspension dynamic deflection. (c) Tire dynamic displacement.

TABLE 2: RMS of the responses.

\begin{tabular}{lccc}
\hline Random excitation & Acceleration of the car body & Suspension dynamic deflection & Tire dynamic displacement \\
\hline Passive suspension & 1.9514 & 0.0302 & 0.0033 \\
Active suspension under optimal parameters & 1.3502 & 0.0219 & 0.0026 \\
Percentage change & $-30.8115 \%$ & $-27.5230 \%$ & $-22.0534 \%$ \\
\hline
\end{tabular}

$\mathrm{B}=0.03$, gs $(t)$ is a continuous piecewise linear function generated by Gaussian white noise, its power is $0 \mathrm{dBW}$, the sampling frequency is (5/s), and the sampling time is $20 \mathrm{~s}$. The time-domain diagram of random excitation is shown in Figure 4 . By substituting the random excitation $x_{d}$ into equation (10), we obtain the following equation:

$$
\begin{aligned}
\dot{x}_{1}= & y_{1}, \\
\dot{y}_{1}= & -\left(x_{1}-x_{2}\right)-\mu\left(x_{1}-x_{2}\right)^{3}-\zeta_{1}\left(y_{1}-y_{2}\right)-\delta\left(y_{1}-y_{2}\right)^{3} \\
& -g x_{1 \tau}+g x_{1}, \\
\dot{x}_{2}= & y_{2} \\
\dot{y}_{2}= & \gamma\left(x_{1}-x_{2}\right)+\gamma \mu\left(x_{1}-x_{2}\right)^{3}+\gamma \zeta_{1}\left(y_{1}-y_{2}\right) \\
& +\gamma \delta\left(y_{1}-y_{2}\right)^{3}-\gamma k\left[x_{2}-B^{*} g s\left(\sqrt{\frac{m_{1}}{k_{1}}} t\right)\right] \\
& -\gamma \zeta_{2}\left[y_{2}-B^{*} \sqrt{\frac{m_{1}}{k_{1}}} \operatorname{dgs}\left(\sqrt{\frac{m_{1}}{k_{1}}} t\right)\right]+\gamma g x_{1 \tau}-\gamma g x_{1},
\end{aligned}
$$

where $B^{*}=(B / L)$.

4.2. Optimization and Simulation. The objective function, constraint conditions, and initial conditions are the same as those under simple harmonic excitation. The optimal feedback parameters of the active suspension system with time-delay feedback control under random excitation are obtained through optimization calculation: $g=-1.10$ and $\tau=0.69$. According to Figure 2, the optimization results meet the stability conditions. The corresponding parameters of the original model are $g_{1}=-19800(\mathrm{~N} / \mathrm{m})$ and $\tau=0.092 \mathrm{~s}$. The body acceleration, suspension dynamic deflection, and tire dynamic displacement of the active suspension system controlled by the optimal feedback parameters and the passive suspension system without control are simulated. The simulation results are shown in Figure 5. In order to quantitatively analyze the damping effect of the active suspension system under optimal parameters control, the RMS values of body acceleration, suspension dynamic deflection, and tire dynamic displacement and their change percentage are listed in Table 2 . The RMS values of car body acceleration, suspension dynamic deflection, and tire dynamic displacement are reduced by $30.8115 \%, 27.5230 \%$, and $22.0534 \%$, respectively. Figure 5 and Table 2 show that under random excitation, the active suspension system with time-delay feedback control also has better damping effect compared with the passive suspension system.

\section{Conclusion}

By adding time-delay displacement feedback control to the (1/4) vehicle nonlinear system, the mathematical model of the time-delay nonlinear control system is obtained. The generalized Sturm criterion and Routh-Hurwitz stability theorem are used to analyze the stability of the system, and 
the critical stability curve of the $g-\tau$ plane is obtained. The multiobjective optimization function is established by considering the body acceleration, suspension dynamic deflection, and tire dynamic displacement. The optimal parameters are obtained by optimizing the parameters of the system under the condition of simple harmonic excitation and random excitation. The conclusions are as follows:

(1) As the control parameters of the time-delay nonlinear active suspension system, the gain coefficient and the time delay affect the system stability. There is a time-delay-independent stability region $g \in$ $(-0.35,0.35)$, in which the system is unconditionally stable. The stability region of the $g \sim \tau$ plane is obtained by stability analysis.

(2) Through the analysis and simulation of the active suspension system under the simple harmonic road excitation, it can be seen that the time-delay displacement feedback control can reduce the vibration of the system, and there are optimal feedback parameters to make the running state of the car optimal. The above conclusions are further verified by the analysis of the active suspension system under the road random excitation. It is proved that the time-delay displacement feedback control is an effective active suspension control method.

(3) The design of multiobjective optimization proposed in this paper can improve ride comfort, driving safety, and handling stability and provide guidance for comprehensively improving vehicle performance.

\section{Data Availability}

The data used to support the findings of this study are available from the corresponding author upon request.

\section{Conflicts of Interest}

The authors declare that they have no conflicts of interest.

\section{Acknowledgments}

This study was supported by the National Natural Science Foundation of China (grant no. 51275280).

\section{References}

[1] R. A. Williams and A. Best, "Control of a low frequency active suspension," in Proceedings of the International Conference on Control, Coventry, UK, March 1994.

[2] Y. M. Rong, Y. W. Wu, and Q. Su, "The research of the strategy in the active suspension of automobile," Machinery Design \& Manufacture, vol. 2005, no. 7, p. 89, 2005.

[3] N. Olgac, D. M. McFarland, and B. T. Holm-Hansen, "Position feedback-induced resonance: the delayed resonator," in Proceedings of the ASME Winter Annual Meeting, vol. 38, pp. 113-119, Anaheim, CA, USA, December 1992.

[4] N. Olgac, H. Elmali, and S. Vijayan, "Introduction to the dual frequency fixed delayed resonator," Journal of Sound and Vibration, vol. 189, no. 3, pp. 355-367, 1996.
[5] N. Jalili and N. Olgac, "Multiple delayed resonator vibration absorbers for multi-degree-of-freedom mechanical structures," Journal of Sound and Vibration, vol. 223, no. 4, pp. 567-585, 1999.

[6] K. A. Alhazza, A. H. Nayfeh, and M. F. Daqaq, "On utilizing delayed feedback for active-multimode vibration control of cantilever beams," Journal of Sound \& Vibration, vol. 319, no. 3-5, pp. 735-752, 2009.

[7] K. Liu, L. X. Chen, and G. P. Cai, "Experimental study of delayed positive feedback control for a flexible beam," Theoretical and Applied Mechanics Letters, vol. 1, no. 6, Article ID 063003, 2011.

[8] Q. Wang, C. Ren, J. Zhou, and L. Zhang, "The double-delay reducing vibration control for five-degree-of-freedom halfvehicle model in idle condition," Journal of Low Frequency Noise, Vibration and Active Control, vol. 39, no. 1, pp. 203215, 2020.

[9] H. Du, J. Lam, and K. Y. Sze, "Design of non-fragile $H \infty$ controller for active vehicle suspensions," Journal of Vibration and Control, vol. 11, no. 2, pp. 225-243, 2005.

[10] H. Du, J. Lam, and K. Y. Sze, "Hodisturbance attenuation for uncertain mechanical systems with input delay," Transactions of the Institute of Measurement and Control, vol. 27, no. 1, pp. 37-52, 2005.

[11] S. Zhou, Y. Li, Z. Ren, G. Song, and B. Wen, "Nonlinear dynamic analysis of a unilateral vibration vehicle system with structural nonlinearity under harmonic excitation," $M e$ chanical Systems and Signal Processing, vol. 116, no. 1, pp. 751-771, 2019.

[12] G. I. Koumene Taffo, M. Siewe Siewe, and C. Tchawoua, "Stability switches and bifurcation in a two-degrees-of-freedom nonlinear quarter-car with small time-delayed feedback control," Chaos, Solitons \& Fractals, vol. 87, pp. 226-239, 2016.

[13] Y. Y. Zhao and J. Xu, "Mechanism analysis of delayed nonlinear vibration absorber," Chinese Journal of Theoretical and Applied Mechanics, vol. 40, no. 1, pp. 98-106, 2008.

[14] Z. H. Wang and H. Y. Hu, "Stability switches of time-delayed dynamic systems with unknown parameters," Journal of Sound and Vibration, vol. 233, no. 2, pp. 215-233, 2000.

[15] R. D. Naik and P. M. Singru, "Resonance, stability and chaotic vibration of a quarter-car vehicle model with time-delay feedback," Communications in Nonlinear Science and $\mathrm{Nu}$ merical Simulation, vol. 16, no. 8, pp. 3397-3410, 2011.

[16] S. I. Niculescu, Delay Effects on Stability: A Robust Control Approach, Springer, London, UK, 2001.

[17] H. Y. Hu and Z. H. Wang, Dynamics of Controlled Mechanical Systems with Delayed Feedback, Springer, Heidelberg, Germany, 2002.

[18] V. B. Kolmanovsk and V. R. Nosov, Stability of Functional Differential Equations, Academic Press, London, UK, 1986.

[19] N. Olgac and R. Sipahi, "An exact method for the stability analysis of time-delayed linear time-invariant (LTI) systems," IEEE Transactions on Automatic Control, vol. 47, no. 5, pp. 793-797, 2002.

[20] J. Xu and Q. S. Lu, "Bifurcations and chaos due to time delay in a delayed control non-autonomous system," Acta Mechanica Sinica, vol. 35, no. 4, pp. 443-451, 2003.

[21] H.-P. Wang, J. Li, and K. Zhang, "Stability and Hopf bifurcation of the maglev system with delayed speed feedback control," Acta Automatica Sinica, vol. 33, no. 8, pp. 829-834, 2007.

[22] R.-H. Huan, L.-X. Chen, and J.-Q. Sun, "Multi-objective optimal design of active vibration absorber with delayed 
feedback," Journal of Sound and Vibration, vol. 339, pp. 5664, 2015.

[23] C. Liu, S. Yue, J. Zhou, and J. L. Zhou, "Piezoelectric optimal delayed feedback control for nonlinear vibration of beams," Journal of Low Frequency Noise, Vibration and Active Control, vol. 35, no. 1, pp. 25-38, 2016.

[24] S. Li, J. L. Zhou, C. B. Ren, and S. Shao, "The research of time delay vibration control with time-varying parameters," Chinese Journal of Theoretical and Applied Mechanics, vol. 50, no. 1, pp. 99-108, 2018.

[25] B. C. Wen, Nonlinear Vibration in Engineering, Chinese Science Press, Beijing, China, 2007. 